\title{
L'EUROPE, ENTRE FOISONNEMENT DES TERRITOIRES ET MODĖLES D'ORGANISATION TERRITORIALE
}

\author{
Par Christophe QUÉVA*
}

Malgré de grandes différences dans leur organisation territoriale, les pays européens se caractérisent par un certain nombre de convergences qui nous invitent à nous interroger sur le rôle que peut jouer l'Union européenne en tant que pourvoyeuse de modèles territoriaux. À cet égard, la politique régionale de cette dernière implique que chaque État membre dispose d'un échelon infranational qui soit à même de gérer et de répartir les aides financières de I'Union, et dans les nouveaux États membres, l'adhésion à l'Union a impliqué la constitution de nouvelles « régions ". À l'échelon local, les réformes territoriales menées dans les anciens comme dans les nouveaux États membres semblent également se caractériser par une certaine convergence vers un modèle de territoire local, élargi par les fusions de communes ou la constitution d'intercommunalités. Nous analyserons dans cet article les enjeux d'un possible modèle européen d'organisation territoriale, en en présentant également les limites dans le cadre d'une Europe marquée par le foisonnement des territoires.

\section{INTRODUCTION}

La devise de l'Union européenne - « Unie dans la diversité » - reflète bien le paradoxe du projet européen : celui de construire un territoire qui ne remette pas en cause la pluralité des États, des régions et des territoires locaux.

À l'échelle européenne, les territoires foisonnent, et l'image française du millefeuille administratif ne saurait suffire à caractériser toute la richesse de la diversité territoriale européenne.

En effet, chaque État, qu'il soit unitaire (comme la majorité des États européens), fédéral (comme l'Allemagne, l'Autriche ou la Belgique) ou régionalisé (comme l'Italie ou l'Espagne) dispose d'une organisation, d'une histoire et de mutations territoriales spécifiques qui rendent particulièrement complexe la construction d'une maille territoriale qui soit commune à l'échelle de toute l'Europe. Néanmoins, l'utilité de cette maille commune se fait sentir, notamment, pour l'attribution des fonds de la politique régionale européenne qui vise à corriger les disparités entre les régions des différents États membres. Dans cette perspective, I'Union européenne pourrait apparaître comme une pourvoyeuse de modèles territoriaux, constituant autant de cadres régulateurs des dynamiques nationales et infranationales.

Un processus d'européanisation se dessine, au sens de "dynamique de similitude qui possède une dimension géographique. [Cette européanisation] permet, en effet, d'envisager les transformations des systèmes territoriaux en Europe, en étudiant les structures spatiales induites par cette dynamique et leur agencement avec les configurations territoriales qui les accueillent » (BOULINEAU et SUCIU, 2008). 
Dans les nouveaux pays membres de l'Union, en particulier dans les pays d'Europe centrale et orientale (PECO), cette européanisation a été synonyme d'un profond bouleversement des structures territoriales avec le passage d'une organisation centralisée, sous les anciens régimes communistes, à une décentralisation couplée à un processus de régionalisation - celle-ci constituant le cadre nécessaire à l'obtention des aides financières de la politique régionale. Dans quelle mesure les convergences territoriales à l'œuvre dans et entre les États européens relèvent-elles ou non d'un modèle commun de réorganisation des territoires ? Cet article a pour objet d'interroger les modèles territoriaux à l'œuvre dans quelques-uns des États européens, ainsi qu'à l'échelle de l'Union européenne prise dans son ensemble, afin de tenter de comprendre les dynamiques territoriales communes qui sont à l'œuvre dans le contexte européen.

\section{LE FOISONNEMENT DES TERRITOIRES EN EUROPE}

Dans la perspective de la réalisation du projet européen, il n'est nullement question de remettre en cause la souveraineté des États ou leur organisation territoriale.

Les différences existant entre les États membres dans ce domaine se font particulièrement sentir dans le domaine de l'aménagement: "Les logiques de l'aménagement du territoire, le fonctionnement institutionnel et ses instruments, ainsi que l'interprétation du terme [aménagement] dépendent largement $d u$ contexte national. Le souci fondamental est à peu près le même partout: promouvoir un développement équilibré du territoire et corriger les disparités entre les régions. Par contre, la manière dont la politique d'aménagement du territoire a été institutionnalisée et la façon dont elle est mise en œuvre varient beaucoup selon les différents pays » (GUDER, 2003). Dans ce cadre, les territoires infranationaux, qui sont la référence pour l'attribution des fonds européens de la politique régionale, correspondent à des structures totalement différentes selon les contextes: il s'agit de collectivités territoriales décentralisées (les régions) dans le cas français, d’États fédérés (les Länder) dans le cadre allemand, ou encore de structures intermédiaires (les Communautés autonomes) en Espagne, chacune de ces structures disposant de compétences et de modalités d'action différentes.

Ces différences d'organisation territoriale pèsent sur la mise en œuvre et l'interprétation des politiques européennes.

En France, malgré une décentralisation qui ne cesse de s'accentuer avec les réformes territoriales de 2002-03 et 2013, la logique de l'État unitaire accorde toujours une place majeure aux politiques nationales d'aménagement (comme le montrent les pôles de compétitivité, la politique de la ville, les zones de revitalisation rurale, etc.).

À l'échelle européenne, ce modèle unitaire d'organisation territoriale s'associe à la défense d'une "Europe des États » (SAINT-OUEN, 1993). Face à ce modèle territorial unitaire, l'Allemagne relève d'une logique différente : celle d'un État fédéral composé de seize États fédérés, les Länder, qui disposent chacun de leur propre constitution, d'une organisation territoriale et d'une politique d'aménagement spécifiques - même si la Réunification et le creusement des disparités entre l'Est et l'Ouest du territoire ont pu conduire à un certain renforcement de l'échelon fédéral (QUÉVA, 2007a). Dans le 
contexte européen, l'Allemagne incarne ainsi une organisation territoriale qui relèverait davantage d'une "Europe des régions". Celle-ci est "devenue une exigence importante des Länder, qui aspirent à jouer le rôle de réels Global Players en raison des profits qu'ils retirent des divers fonds structurels de l'Union européenne » (MARTENS, 2003). Les Länder ont d'ailleurs pesé de tout leur poids pour que soit affirmé le principe de subsidiarité, et c'est sous l'impulsion du gouvernement fédéral allemand pressé par les Länder que le Comité des régions a été revalorisé lors de la réunion du Conseil européen d'Amsterdam de juin 1997.

Dans une configuration intermédiaire entre le modèle fédéral et le modèle unitaire, les Communautés autonomes espagnoles s'inscrivent plutôt dans la perspective d'une "Europe des régions", dont les enjeux seraient ici également géopolitiques, au regard du poids de "régions » identitaires aux revendications autonomistes parfois très marquées, comme la Catalogne ou le Pays Basque (BoIX et MAJOR, 2013 ; QUÉVA et VERGNAUD, 2009). Néanmoins, au-delà des différences nationales, la définition d'une échelle d'action européenne - notamment dans le cadre de la politique régionale européenne - implique d'appréhender chacun des États membres au prisme d'une même grille de lecture des territoires.

\section{VERS UN MODĖLE EUROPÉEN D'ORGANISATION ET DE RECOMPOSITION TERRITORIALES ?}

La Commission européenne a ainsi mis en œuvre un découpage territorial destiné à faciliter les comparaisons entre les pays et entre les régions au sein du territoire de I'Union. II s'agit de la nomenclature des unités territoriales statistiques (NUTS). Celleci se fonde sur un découpage de chaque pays en niveaux de collecte statistique correspondant à des seuils de population (1) et recoupant le plus souvent des subdivisons administratives infranationales. Les trois principaux niveaux de maille sont les suivants: NUTS 0 (États), NUTS 1 (grandes régions socio-économiques) et NUTS 2 (régions de base pour l'application des politiques régionales). Ce découpage sert à la fois à la collecte des statistiques mesurant les disparités régionales et à la définition des politiques régionales de l'Union européenne. La figure 1 permet de comparer les niveaux NUTS 1 et NUTS 2 de quatre des plus anciens pays membres de l'Union européenne (l'Allemagne, l'Espagne, la France et l'Italie) et de trois membres récents (la Hongrie et la Pologne, entrées dans l'UE en 2004, et la Bulgarie, entrée en 2007).

Figure 1. Le découpage territorial de I'Union européenne en NUTS 1 et 2 : 
quelques exemples nationaux.

\begin{tabular}{|c|c|c|}
\hline & NUTS 1 & $\begin{array}{c}\text { NUTS } 2 \\
\text { (Politique régionale européenne) }\end{array}$ \\
\hline Allemagne & 16 Länder & 41 Regierungsbezirke (districts) \\
\hline Bulgarie & 2 régions économiques & $\begin{array}{c}6 \text { régions de planification (groupes } \\
\text { de régions) }\end{array}$ \\
\hline Espagne & $\begin{array}{c}7 \text { Agrupacion de comunidades } \\
\text { autonomas }\end{array}$ & 17 comunidades autonomas \\
\hline France métropolitaine & $\begin{array}{l}8 \text { zones économiques } \\
\text { d'aménagement du territoire (ZEAT) }\end{array}$ & 22 régions \\
\hline Hongrie & 3 régions statistiques & $\begin{array}{l}7 \text { groupes de comitat (régions } \\
\text { économico-statistiques) }\end{array}$ \\
\hline Italie & 11 gruppi di regioni & 20 regioni \\
\hline Pologne & 6 régions (regiony) & 16 voïvodies \\
\hline
\end{tabular}

Source : Eurostat, 2013.

Ces NUTS peuvent être interprétés comme la recherche d'une maille territoriale homogène à l'échelle de toute l'Union européenne. En effet, l'idée est de parvenir à une grille de lecture commune, qui passe outre les différences d'organisation territoriale existant entre les États membres.

Signalons par ailleurs que cette nomenclature a parfois pesé sur le découpage territorial des pays candidats à l'intégration dans l'Union européenne. De fait, en aval de ce découpage en NUTS, l'obtention des financements se fonde sur l'existence de collectivités territoriales à l'échelon régional qui soient à même de gérer et de répartir ces financements. Dans ce contexte, selon E. Boulineau (2006), les « demandes de l'Union européenne " en matière statistique peuvent être entendues "comme l'expression d'un "modèle" européen régional ", notamment dans le cas de la Bulgarie, mais aussi, plus généralement, dans celui des PECO (MARCOU, 2002 ; REY et al., 2004).

Ce modèle européen régional rejoint par ailleurs la logique d'un autre modèle : celui du fédéralisme allemand. En effet, "les pays candidats à l'entrée dans l'Union européenne ont mené des réformes territoriales pour constituer des entités administratives au plan régional, condition sine qua non pour l'obtention des financements de l'Union européenne. En ce sens, les pays candidats s'inspirent très souvent, pour leurs réformes territoriales, du modèle fédéral allemand " (MARTENS, 2003).

Dans chaque État membre, les politiques régionales européennes ont dès lors été synonymes d'un redécoupage des territoires nationaux et d'une régionalisation.

Celle-ci s'est parfois articulée autour de collectivités territoriales existantes, mais dans d'autres cas, ce découpage régional s'est parfois avéré quelque peu artificiel : ainsi, dans les PECO, "les nouvelles régions n'ont pas pour l'heure conquis une assise 
politique, opérationnelle et identitaire stabilisée. Elles souffrent d'un certain nombre de faiblesses et cherchent encore leur place à côté des États. Le rôle central des régions consiste aujourd'hui à mettre en œuvre les stratégies de développement territorial financé par les fonds européens » (COUDROY DE LILLE, 2005).

La constitution d'un découpage territorial homogène à l'échelle de l'Union européenne se heurte également à une grande diversité dans les tailles et dans les statuts de ces unités, notamment à l'échelon d'application de la politique régionale (NUTS 2). Ainsi, comme le montre la Figure 2, le maillage en NUTS 2 est très fin en Allemagne ou au Royaume-Uni, où il correspond à une maille infrarégionale, alors qu'il correspond, en France ou en Espagne, à l'échelon régional et, dans certains cas (notamment dans les pays baltes), à l'échelon national.

Figure 2. Le découpage régional de l'Union européenne en NUTS 2 en 2013 


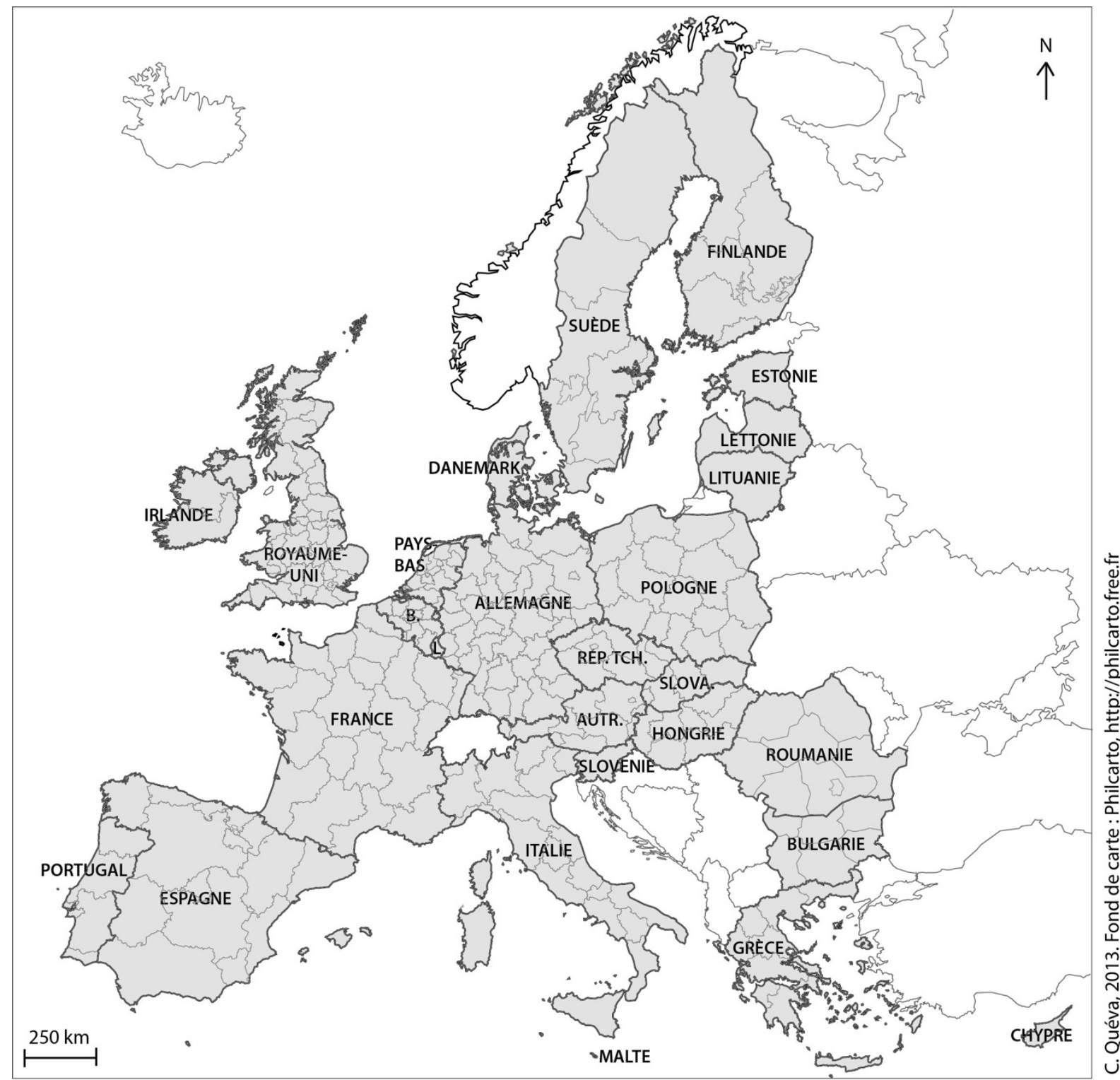

Territoires de I'Union européenne (UE) en 2013*

Territoires hors de I'UE en 2013*

FRANCE Niveau NUTS 0 : territoires des États membres de l'Union europénne

Territoires des États non membres de l'UE

Niveau NUTS 2 : territoires " régionaux »,

bénéficiant des fonds européens de la

politique régionale

* La carte ne fait pas figurer la Croatie, entrée dans l'Union européenne le 1er juillet 2013

Ces différences de maillage constituent des enjeux majeurs, non seulement sur le plan des statistiques nécessaires à l'analyse des disparités régionales (GRASLAND, 2004), mais aussi sur les plans politique et financier, dans le cadre d'une politique régionale européenne fondée sur des types de "régions " totalement différents selon les divers cadres nationaux.

Le découpage en NUTS se voit ainsi critiqué du fait qu'il est associé, selon C. Grasland et G. Hamez (2005), à « une vision statique et atomiste (...), où 200 régions flottent, 
isolées les unes des autres, dans un univers statistique abstrait », sans correspondre à un type identique de collectivité territoriale.

II n'en reste pas moins qu'au-delà de ces découpages régionaux liés à la politique régionale européenne, un certain nombre de convergences territoriales se retrouvent dans les différents pays européens, notamment à l'échelon local.

Ainsi, beaucoup de ces pays ont vu se multiplier chez eux des réformes territoriales valorisant l'idée de territoires locaux élargis grâce à la fusion de communes ou à la constitution d'intercommunalités.

Ainsi, en France, les structures intercommunales occupent désormais une place centrale dans l'architecture territoriale, sans que les communes elles-mêmes aient connu de véritable réforme.

À l'inverse, l'intercommunalité occupe en Allemagne une place très secondaire, du fait de l'importance des fusions intercommunales et des remaniements territoriaux, et ce à l'Est comme à l'Ouest du territoire fédéral (QUÉVA, 2007a).

Dans les deux cas, c'est bien, toutefois, le modèle d'un territoire local élargi qui explique les réformes territoriales mises en œuvre - un modèle de grande commune fonctionnelle que l'on retrouve en Scandinavie.

Enfin, dans le contexte des pays d'Europe centrale et orientale, les réformes menées au niveau local (qui ont été engagées dès le début des années 1990) relèvent d'une forme de mimétisme vis-à-vis des anciens États membres, voire d'un transfert de modèle : en effet, les réformes territoriales locales s'y sont vues encadrées sous la forme de parrainages par les pays occidentaux (la France, l'Allemagne, ou encore les pays scandinaves).

Un certain modèle d'organisation territoriale locale semble ainsi se dessiner en Europe, même si de toute évidence ce modèle s'applique selon des modalités différenciées suivant les contextes nationaux, en fonction de l'héritage historique propre à chaque pays et de son organisation territoriale. Ce modèle reposerait sur une certaine autonomie communale (ou intercommunale), sur la démocratie locale et sur une certaine fonctionnalité spatiale, incarnée par les fusions et les coopérations (inter)communales (QUÉVA, 2007b).

\section{CONCLUSION}

Ainsi, le rôle de l'Union européenne dans la recomposition territoriale des pays membres reste ambigu. Les modalités du découpage territorial relèvent bien de chaque État et non de l'Union européenne en tant que telle, même si celle-ci peut donner certaines orientations en matière d'organisation territoriale, et ce à différents niveaux, à travers l'idée d'une fédération d'États nations, de "régions » de niveau NUTS 2 capables de prendre en charge la politique régionale, ou encore d'espaces (inter)communaux élargis et fonctionnels. Certes, un certain nombre de convergences entre les pays européens semblent se dessiner à l'échelon local dans l'optique d'États largement décentralisés et régionalisés, et ces convergences semblent conforter l'idée 
qu'est en train de se constituer un modèle européen de recomposition territoriale, dans les anciens comme dans les nouveaux pays membres de l'Union européenne. Néanmoins, les différences nationales pèsent encore suffisamment pour qu'il soit nécessaire de nuancer la prégnance de ce modèle, qui relève avant tout des discours et des représentations, ainsi que de jeux de pouvoirs valorisant avant tout les États et leurs territoires nationaux. 


\section{NOTES}

* Université Paris 1 Panthéon-Sorbonne, UMR Géographie-Cités 8504.

Courriel : christophe.queva@univ-paris1.fr

(1) NUTS 1: de 3 à 7 millions d'habitants en moyenne vivent dans les unités territoriales statistiques de ce type dans le pays correspondant (pour les NUTS 2, ce nombre varie de 800000 à 3000000 d'habitants). 


\section{BIBLIOGRAPHIE}

BOIX (C.) \& MAJOR (J.-C.), « La marche de la Catalogne vers l'autodétermination », Politique étrangère, 4, pp. 37-49, 2013.

BOULINEAU (E.), " Au seuil de l'Europe, la difficile régionalisation de la Bulgarie », in BLETON-RUGET (A.), COMMERÇON (N.) \& GONOD (P.) (dir.), Territoires institutionnels, territoires fonctionnels, pp. 209-217, 2006.

BOULINEAU (E.) \& SUCIU (M.), « Décentralisation et régionalisation en Bulgarie et en Roumanie. Les ambiguïtés de l'européanisation ", L'Espace géographique, 4, pp. 349-363, 2008.

COUDROY de LILLE (L.), « Une nouvelle architecture territoriale pour les États d'Europe centrale et orientale », Géoconfluences, 2005.

http://geoconfluences.ens-lyon.fr/geoconfluences/doc/etpays/Europe/EurScient.htm

GRASLAND (C.) \& HAMEZ (G.), « Vers la construction d'un indicateur de cohésion territoriale européen ? », L'Espace géographique, 2, pp. 97-116, 2005.

GRASLAND (C.), "Les inégalités régionales dans une Europe élargie », in CHAVANCE (B.) (dir.), Les Incertitudes du grand élargissement. L'Europe centrale et Balte dans l'intégration européenne, pp. 181-214, 2004.

GUDER (U.), L'aménagement du territoire et la politique régionale en Allemagne : vers une européanisation en douceur?, Groupe d'Études et de Recherches «Notre Europe », 54 p., 2003.

MARCOU (G.), « L'adaptation des structures territoriales face à la politique régionale communautaire », Revue d'études comparatives Est-Ouest, vol. 32, n³, pp. 131-167, 2002.

MARTENS (S.), « Le fédéralisme allemand: modèle pour le fédéralisme européen ? », L'Allemagne d'aujourd'hui, 164, pp. 22-37, 2003.

QUÉVA (C.), « Les paradoxes de la Région en Allemagne, entre réseaux et territoires : la région, outil de déterritorialisation? », Annales de géographie, 653, pp. 41-64, 2007a.

QUÉVA (C.), "Entre territoires et réseaux : la requalification des espaces locaux en France et en Allemagne. Jeux d'acteurs, d'échelles et de projets », Thèse de doctorat de géographie (dir. Gérard Di Méo), Université de Bordeaux 3, 2007b.

QUÉVA (C.) \& VERGNAUD (G.), « Du territoire à l'intermédiarité : essai de réflexions croisées sur les constructions territoriales locales en Allemagne, en France et en Espagne ", in VANIER (M.) (dir.), Territoires, territorialité, territorialisation. Controverses et perspectives, pp. 141-150, 2009. 
REY (V.), COUDROY de LILLE (L.) \& BOULINEAU (E.) (dir.), L'Élargissement de l'Union européenne: réformes territoriales en Europe centrale et orientale, 246 p., 2004.

SAINT-OUEN (F.) (1993), « De l'Europe des États à l'Europe des régions », Relations internationales, 73, pp. 81-94, 1993. 